\title{
Sedentarismo y actividad física en los estudiantes de la universidad estatal península de Santa Elena
}

Belén Narcisa Quirumbay Vera

belen.quirumbayvera@upse.edu.ec

Nallely Brigith Pazmiño Mantuano nallely.pazminomantuano@upse.edu.ec

Yamilet Elena García Chila

yamilet.garciachila@upse.edu.ec

Juliana Nerexy Rodríguez Domínguez juliana.rodriguezdominguez@upse.edu.ec

Dania Fernanda Savedra Toala dania.savedratoala@upse.edu.ec

Karla Arianna Medina Cuesta karla.medinacuesta@upse.edu.ec

Yajaira Maribel Muentes Hidalgo jajaira.muenteshidalgo@upse.edu.e

Adriana Angeline Rosado Yagual adriana.rosadoyagual.@upse.edu.ec

Universidad Estatal Península de Santa Elena La Libertad - Ecuador

\section{RESUMEN}

El presente estudio tiene por objetivo: Diagnosticar el nivel de sedentarismo y de actividad física en los estudiantes de la Carrera de Educación Inicial de la Universidad Estatal Península de Santa Elena, Santa Elena - Ecuador. La muestra estuvo constituida por 205 estudiantes cuyas edades oscilan entre los 27 y 41 años. Se empleó un enfoque cuantitativo con una metodología descriptiva de corte transversal. Se aplicó como instrumento la encuesta, validada bajo juicio de expertos. Mediante, el coeficiente de Cronbach se garantiza el índice de confiabilidad arrojando un valor de 0.764 que 
determina una confiabilidad aceptable. Los resultados más relevantes indican que, el 22\% de los estudiantes desarrolla algún tipo de actividad física, mientras que, el 78\% las realiza con baja frecuencia manteniendo un comportamiento sedentario. Se concluye que, el sedentarismo es una conducta bastante frecuente en los estudiantes universitarios asociado al escaso interés hacia la práctica de la actividad física, por lo que es necesario implementar nuevas estrategias que animen a los estudiantes a desarrollar estilos de vida más saludables.

Palabras clave: sedentarismo; actividad física; estudiantes 


\title{
Sedentarism and physical activity in the students of the Santa Elena peninsula state university
}

\begin{abstract}
The objective of this study is to: Diagnose the level of sedentary lifestyle and physical activity in the students of the Initial Education Career of the Santa Elena Peninsula State University, Santa Elena - Ecuador. The sample consisted of 205 students whose ages range between 27 and 41 years. A quantitative approach was used with a descriptive cross-sectional methodology. The survey was applied as an instrument, validated under expert judgment. Through the Cronbach coefficient, the reliability index is guaranteed, yielding a value of 0.764 , which determines an acceptable reliability. The most relevant results indicate that $22 \%$ of the students develop some type of physical activity, while $78 \%$ do it infrequently, maintaining a sedentary behavior. It is concluded that sedentary lifestyle is a fairly frequent behavior in university students associated with little interest in the practice of physical activity, so it is necessary to implement new strategies that encourage students to develop healthier lifestyles.
\end{abstract}

Keywords: sedentary lifestyle, physical activity, students

Artículo recibido: 02 enero 2022

Aceptado para publicación: 28 enero 2022

Correspondencia: belen.quirumbayvera@upse.edu.ec

Conflictos de Interés: Ninguna que declarar 


\section{INTRODUCCIÓN}

El estudio se articula a la asignatura Diseño de Proyectos Educativos que se imparte en el sexto semestre de la Carrera de Educación Inicial de la Universidad Estatal Península de Santa Elena, donde surge la necesidad de Diagnosticar el nivel de sedentarismo y de actividad física en los estudiantes de los primeros semestres de la Carrera antes mencionada. El comportamiento sedentario se ha visto evidenciado con más frecuencia en la actualidad, precisamente por la crisis sanitaria que atraviesa el mundo como resultado de la pandemia generada por el Covid-19.

De acuerdo a Márquez et al. (2006) "Durante la última década se han realizado investigaciones en torno al sedentarismo por ser considerado un factor de riesgo para incrementar el padecimiento de afectaciones en la salud de las personas" (2006, pp. 1224). Referente a eso, se considera a los jóvenes universitarios un grupo vulnerable para el desarrollo de la conducta sedentaria debido a la escasa actividad física que practican por enfocarse en su rendimiento académico, en consecuencia, están expuestos a padecer enfermedades relacionadas a la insuficiente actividad física.

El estilo de vida sedentario, frecuentemente, se mantiene no solo durante las etapas de formación intelectual, sino también, en la futura vida profesional, esto es parte de aproximadamente el 75\% de la población mundial (Navarrete et al, 2019, pp. 46-52). En efecto, se observa la poca importancia de mantener una vida activa y generar el hábito de realizar actividades físicas con la finalidad de evitar el sedentarismo y los factores de riesgo que esto conlleva.

Es claro que, el comportamiento sedentario afecta al bienestar físico y mental de las personas; así mismo, la práctica de actividades físicas lo mejora. Por ello, la Organización Mundial de la Salud en el 2020, establece directrices sobre la importancia que tiene desarrollar actividades físicas:

Las nuevas directrices recomiendan por lo menos de 150 a 300 minutos de actividad física aeróbica de intensidad moderada o vigorosa por semana para todos los adultos, incluidas las personas que viven con afecciones crónicas o discapacidad, y un promedio de 60 minutos al día para los niños y adolescentes. (Organización Mundial de la Salud, 2020) 
Tanto para niños, adolescentes y adultos, la actividad física es mucho más beneficiosa que permanecer totalmente inactivo, por lo que, ir agregando en la rutina diaria pequeñas dosis de actividad física y posteriormente cumplir con el tiempo adecuado según las recomendaciones de la OMS evitará la vida sedentaria. En los jóvenes universitarios debe surgir el interés propio y la organización del tiempo para cumplir con tales directrices y así optimizar su bienestar físico y mental.

El término sedentario proviene del lat., sedentarius que trabaja sentado, der. de sedere estar sentado" (Real Academia Española, 2020). Esto, se corrobora con lo señalado por la Organización Mundial de la Salud (OMS) que define sedentario como "la poca agitación o movimiento" (Vélez et al, 2014, p. 306). Es decir, un individuo es sedentario cuando lleva un estilo de vida donde desarrolla una escasa actividad física, de tal manera que, en sus actividades cotidianas la mayor parte de su tiempo pasa sentado o en reposo. El sedentarismo es definido como "actividades asociadas a un gasto energético e incluye actividades como estar sentado, ver televisión, conducir" (Leiva et al, 2017, p. 459). Uno de los factores que propicia a llevar una vida sedentaria es, la falta de tiempo para asistir a programas orientados al desarrollo de actividades físicas, de igual modo, usar un aparato tecnológico en el hogar, trabajo o escuela, también, "las sociedades actuales se caracterizan por sustituir el tiempo libre dedicado a la práctica de actividad física por el uso de tecnologías, lo que recientemente se ha denominado como sedentarismo tecnológico" (Rivera et al, 2018, pp. 17-18).

Cuando una persona no realiza ejercicio físico pierde masa muscular, también, se encuentra cansado, su sistema inmunológico está débil y con frecuencia presenta problemas de sueño debido a la inactividad. Indica Domínguez et al. (2020), que la actividad física es el movimiento del cuerpo que es producido por los músculos, produciendo un gasto energético durante un período determinado, es decir, es el esfuerzo que se tiene para lograr medir la fuerza muscular.

"El cuerpo del ser humano está preparado para moverse, más aún, tiene la necesidad de hacerlo. No obstante, a pesar de esta necesidad, la práctica de actividad física en la población es cada vez menos frecuente" (Escalante, 2011, p. 325). Es decir, en la actualidad los profesionales de diferentes áreas del conocimiento desarrollan el hábito sedentario, ya que, la mayor parte del tiempo se encuentran obligados a estar frente a un computador; así mismo, los estudiantes universitarios tras el inicio de la Pandemia y con 
el cambio de la modalidad presencial a la virtualidad, también, pasan la mayor parte de su tiempo frente a una pantalla, lo cual es un limitante para realizar actividades físicas. De acuerdo a Escalante (2011), la actividad física se define como cualquier movimiento corporal producido por la contracción de un músculo esquelético que genera un gasto de energía, y se ubica en la lista de acciones protectores más importantes para prevenir muchos problemas crónicos de los que se ven afectadas varias personas en todo el mundo (pp. 325-328). Con el desarrollo tecnológico, la expansión de los procesos de globalización, se observa una tendencia menor en actividad física e incremento del sedentarismo, según la Escuela Nacional de Salud y Nutrición (2013), en las investigaciones realizadas en la actualidad el sedentarismo es el cuarto factor de riesgo, asociado con el $6 \%$ de la mortalidad a escala mundial, seguida de la hipertensión arterial, consumo de cigarrillo y niveles altos de glucosa (pp. 79-82), siendo el sedentarismo preocupante para cualquier estilo de vida.

Por otro lado, Claros et al. (2011), menciona que, a lo largo de los siglos la actividad física es una promoción de la salud que contribuye a construir resultados para el mejoramiento de una buena calidad de vida, porque esta ayuda a prevenir la obesidad (pp. 104-105). En la actualidad, las acciones de promoción de actividad física tienen en cuenta que existen varios factores que permiten que las personas no la ejecuten por la falta de ganas, desinterés, motivación o escaso tiempo.

Hoy en día, expertos coinciden en que la mejor medicina es la prevención, en este sentido, los médicos confirman la importancia de cambiar estilos de vida incompatibles con la salud como la principal estrategia de vida (Cala y Navarro, 2011, p. 2). Practicar actividad física se refiere al desempeño de todas las tareas en las que el cuerpo utiliza energía, y es importante realizarlas periódicamente, esto debido a que:

"El descenso en el nivel de aptitud física de las poblaciones humanas en todo el mundo aumenta el predominio de la mortandad precoz causada por enfermedades de la 'civilización', demostrando que el sedentarismo, como estilo de vida, puede ser nocivo para el individuo y potencialmente dañoso para la sociedad" (Matsudo, 1997, citado por, Bastos et al, 2005).

Siendo así, el sedentarismo es un mal predominante en la época actual y no solo afecta como individuos sino como sociedad, sin embargo, depende de cada ser humano tener 
como propósito cambiar este estilo de vida, si desea mantener un ritmo de vida saludable. En torno a los beneficios que conlleva realizar actividad física se distinguen tales como el simple hecho de aprender a seguir reglas, aumentar habilidades motoras que implica el favorecimiento del crecimiento de músculos y huesos, así mismo disminuye el riesgo de obesidad (Perea et al, 2019, pp. 121-125). Hacer que el cuerpo se encuentre en movimiento implica una mejoría en la salud en general, por consiguiente, los estudiantes precisamente universitarios tienen mejor rendimiento académico porque desarrollan más las habilidades físicas y mentales.

El rendimiento académico se asocia a la actividad física debido a los múltiples beneficios que ofrece como la disminución de estrés, ansiedad, o depresión. De acuerdo a Luque et al (2019), para obtener una influencia positiva en el rendimiento académico, es necesario realizar actividad física un tiempo estimado entre 30-90 minutos diarios, consiguiendo que el alumno mejore en su atención y capacidad de resolución de problemas (Luque et al. 2019, p.100), es decir, realizar actividad física ayuda al rendimiento cognitivo, los estudiantes al estar activos mejoran su concentración dando paso a adquirir nuevos hábitos beneficiosos para la mejora de su rendimiento académico y por ende para su vida. Ante lo expuesto, el presente estudio tiene como objetivo de investigación: Diagnosticar el nivel de sedentarismo y actividad física en muestra de estudiantes de la Carrera de Educación Inicial de la Universidad Estatal Península de Santa Elena.

\section{ESTRATEGIAS METODOLÓGICAS O MATERIALES Y MÉTODOS}

La presente investigación se abordó bajo un enfoque cuantitativo que "se centra en la teoría sustantiva del problema a investigar" (Sautu, 2005, p. 46). Es decir, los investigadores se separan del problema de estudio para poder generar a partir de esto conocimientos objetivos sobre él; se ha utilizado en esta investigación con la finalidad de exponer, pronosticar, indagar o describir a través de datos cuantificables la razón de ser de la problemática demostrando con el uso de la estadística patrones de comportamiento de los estudiantes universitarios con respecto a la temática del sedentarismo y la actividad física.

El alcance de esta investigación es descriptivo debido a que, "comprende la descripción, registro, análisis e interpretación de la naturaleza actual y la composición de los fenómenos" (Tamayo \& Tamayo, 2003, p. 46). De este modo, se utiliza la recolección de datos para probar hipótesis con base en la medición numérica y el análisis estadístico, se 
emplea este tipo de investigación para conocer y definir características del nivel de sedentarismo y de actividad física en los estudiantes universitarios, a través de medición de datos para así lograr determinar las condiciones reales del estudio, correlacionando la información.

\section{El estudio presenta un corte transversal el cual se define como:}

El diseño es rápido, económico y permite el cálculo directo de la prevalencia de una condición. Además, la relación de temporalidad entre la exposición y el efecto son medidas de forma simultánea en un único período, no siendo posible identificar una direccionalidad en la temporalidad (Cvetkovic et al, 2021, p. 179).

Por su característica observacional se emplea el corte transversal en la investigación descriptiva, además, implica la recolección de datos en un solo corte en el tiempo con la encuesta como instrumento.

La muestra estuvo constituida por 205 estudiantes universitarios cuyas edades oscilan entre los 17 y 41 años, ellos pertenecen a primero, segundo, tercero y cuarto semestre de la Carrera de Educación Inicial de la Universidad Estatal Península de Santa Elena.

Para la validez del instrumento se plantea el juicio de expertos, este se define como "una opinión informada de personas con trayectoria en el tema, que son reconocidas por otros como expertos cualificados en éste, y que pueden dar información, evidencia, juicios y valoraciones" (Escobar \& Martínez, 2008, p. 29). La investigación enfoca características como claridad, entendible y de fácil comprensión; relación, se adapta al contexto estudiado; coherencia, se relaciona directamente con las dimensiones expuestas.

La confiabilidad del instrumento se obtuvo con el programa estadístico SPSS con un valor de Alpha de Cronbach de 0,764 que lo determina como aplicable y se encuentra dentro del rango para establecer la confiabilidad del instrumento, teniendo como referencia:

El valor mínimo aceptable para el coeficiente Alfa de Cronbach es 0,70 por debajo de ese valor la consistencia interna es baja; por su parte, el valor máximo es 0,90 por encima de ese valor se considera redundancia o duplicación (Cortina, 1993 citado por Oviedo \& Campo, 2005, p.577). Como instrumento para la recolección de datos se elaboró una encuesta con 16 ítems con su respectiva escala de Likert misma que se le consignó los siguientes valores: nunca (1), raramente (2), ocasionalmente (3), frecuentemente (4) y muy frecuente (5). 
El instrumento estuvo conformado por tres (3) dimensiones: Interés hacia la actividad física; Organización del tiempo para el desarrollo de la actividad física y; Desarrollo de la actividad física.

Los datos se procesaron en el programa estadístico SPSS, sus valores se analizan con medidas de tendencia central (media, mediana y moda) y la desviación estándar, por lo que existe la oportunidad de obtener intervalos para determinar el grado de cumplimiento de cada indicador del instrumento.

\section{RESULTADOS Y DISCUSIÓN}

En las siguientes tablas se expresan los resultados del instrumento aplicado a los estudiantes de la Carrera de Educación Inicial de la Universidad Estatal Península de Santa Elena con el propósito de conocer el interés hacia la actividad física, el nivel de actividad física realizado y, la organización del tiempo respecto a las actividades académicas para realizar la actividad física.

Gráfico 1. Interés de los estudiantes hacia la actividad física

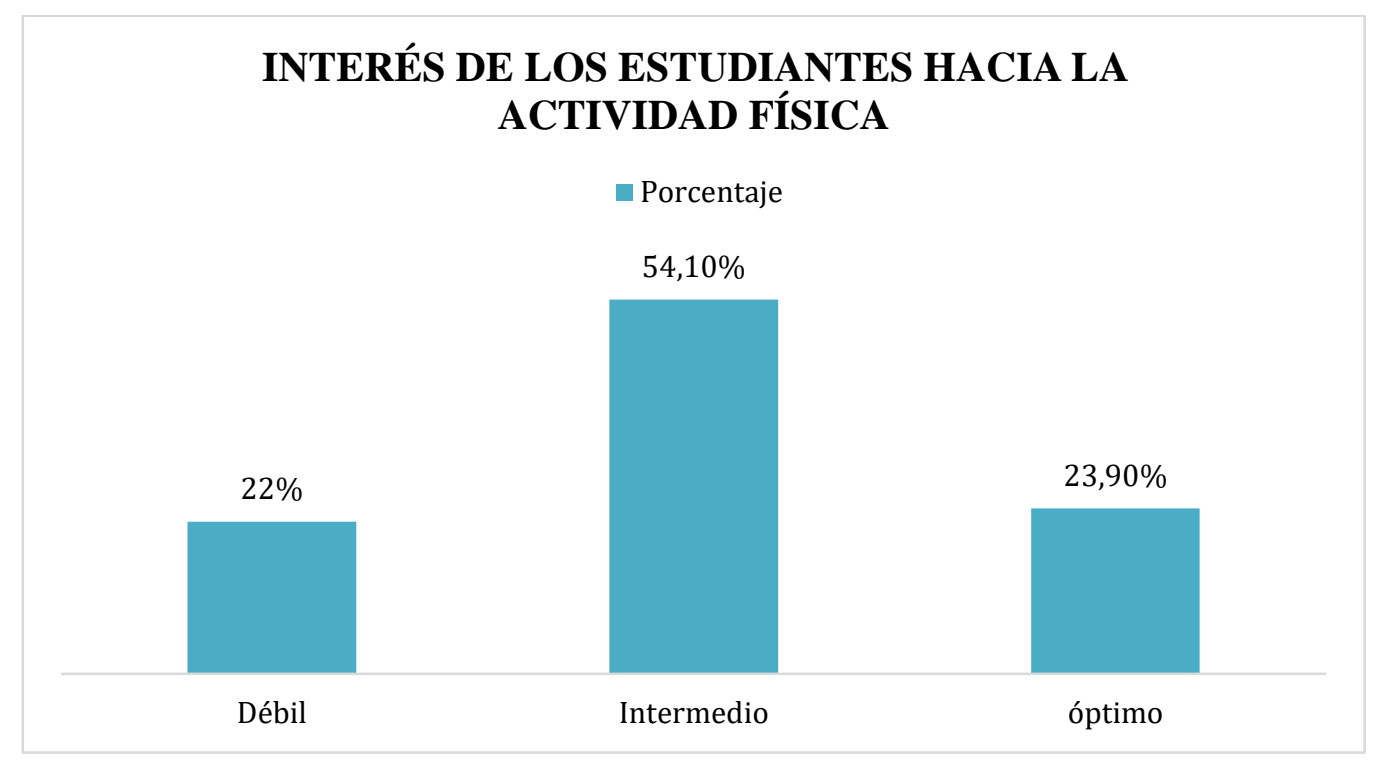

Nota: Encuesta estudiantes de la Carrera de Educación Inicial

Se observa en el gráfico 1, que del total de estudiantes encuestados el $22 \%$ presenta un interés débil hacia la actividad física; esto se debe, a lo mencionado por Ruis y Cols (2001), “en la población universitaria decrece el interés por la práctica de actividades físico-deportivas como consecuencia de la aparición de nuevos intereses"

Es decir, los intereses que tienen los estudiantes universitarios se modifican con el tiempo, donde suelen pasar ocupados y/o preocupados por terminar sus deberes, olvidando la 
importancia que tiene realizar actividad física, para mantener un equilibrio en su vida.

El 54\% de estudiantes se encuentra en el rango intermedio, esto quiere decir, que, existe un interés moderado hacia la actividad física que no siempre se materializa con el desarrollo de actividades que tengan un impacto positivo en la salud. Herrera et al. (2012) plantea que, "existen diversas opiniones acerca de que es la calidad de vida y cómo alcanzarla, para algunos significa ausencia de problemas de salud; para otros, es tener abundancia de tiempo libre para disfrutar de lo que deseen hacer"; los estudiantes universitarios muestran motivación que probablemente nace del conocimiento de los beneficios para su salud, pero para que sea un estilo de vida saludable deben organizar su tiempo, de tal manera, que, en su rutina exista una hora específica de actividad física.

El 23,9\% de estudiantes se encuentra en el rango óptimo por lo cual se infiere que presentan un interés fuerte hacia el desarrollo de la actividad física. Castañeda-Vázquez et al. (2016) argumenta que "diversos estudios avalan los numerosos beneficios que conlleva la práctica regular y adecuada de actividad física sobre diferentes dimensiones de la salud personal, física y psicológica". Los estudiantes universitarios ubicados en este rango palpan la relación que tiene la actividad física y la salud, de alguna manera, fomentan hábitos y estilos de vida saludables, ayudándolos a rendir mejor en sus estudios, trabajo y rutina diaria, culminando con éxito su día.

Gráfico 2. Organización del tiempo para desarrollar la actividad física

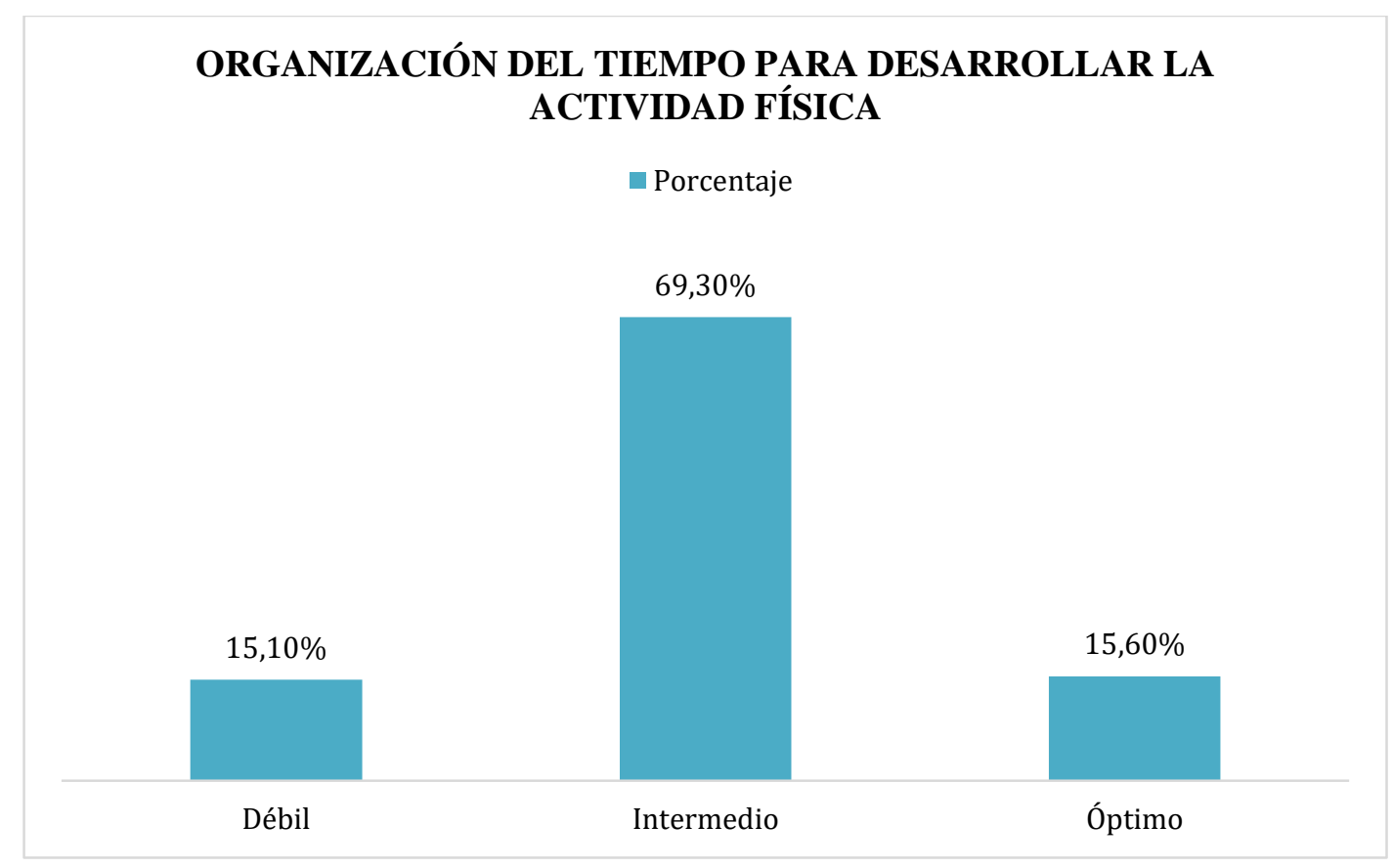

Nota: Encuesta estudiantes de la Carrera de Educación Inicial 
En el gráfico 2 de Organización del tiempo para desarrollar actividad física, se refleja un porcentaje débil del $15 \%$ que concierne a que presenta falencias de organización de su tiempo, por "la escasez o falta de adecuación de las actividades deportivas planificadas en este grupo de población pueden dificultar que los estudiantes continúen asiduamente la práctica de actividad física en la etapa universitaria" ( Rona y Gokmen, 2005, como se citó en Práxedes et al., 2016, p. 124), esto demuestra la poca distribución de actividades atléticas en el diario vivir del estudiante.

Por otro lado, está el 69\% del ítem intermedio demuestra que los universitarios, aun teniendo organización de su tiempo esta no es llevada a la acción correctamente, como lo explica según Rivera et al. (2018) "la población moderna dedica significativas horas de tiempo libre al uso de aparatos electrónicos" (pp.17-18), lo que garantiza menos movilidad y probabilidad de mantener un peso adecuado y con mayor probabilidad de contraer enfermedades relacionadas al sedentarismo.

El 15\% de los estudiantes presenta una óptima organización en sus actividades cotidianas y académicas según Álvarez et al. (2014) "En la práctica deportiva se potencian muchas habilidades y competencia de relevancia para el desarrollo personal y la integración sociolaboral" (p.70), además, de obtener una vida saludable y aportando por ende su buen estado de salud, fomenta en esta población una mejor resolución de problemas.

Gráfico 3. Desarrollo de la actividad física de los estudiantes

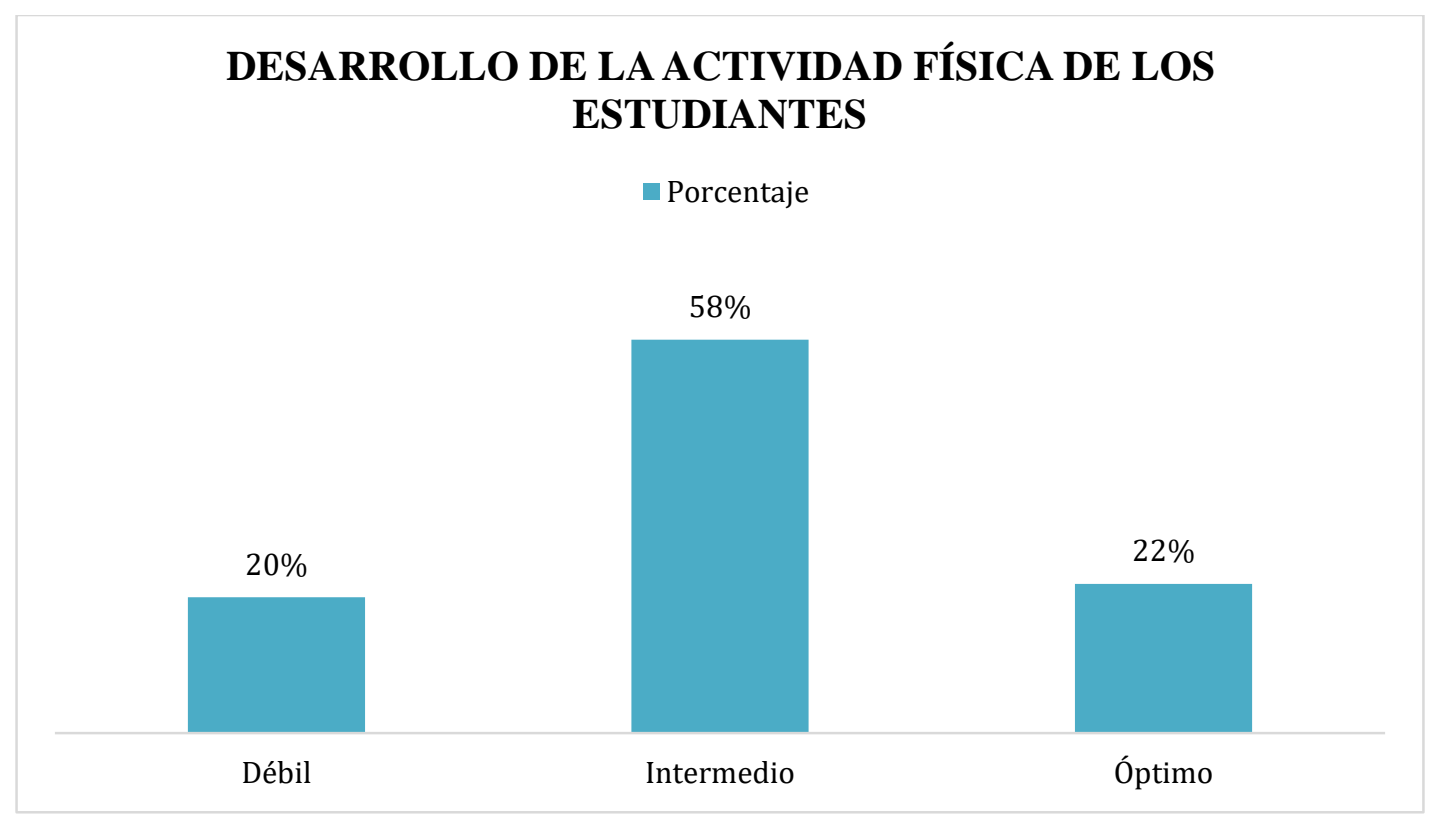

Nota: Encuesta estudiantes de la Carrera de Educación Inicial 
Se evidencia en la tabla 3 , el desarrollo de los estudiantes de la actividad física que del total de la muestra el $20 \%$ presenta un interés débil hacia el desarrollo de la actividad física, esto se debe a lo mencionado por Rivera et al (2018) "las sociedades actuales se caracterizan por sustituir el tiempo libre dedicado a la práctica de actividad física por el uso de tecnologías, lo que recientemente se ha denominado como sedentarismo tecnológico" esto se debe a que en la actualidad las personas no desarrollan actividad física ya que utilizan sus tiempos libres para realizar otras actividades que podrían perjudicar su vitalidad y/o salud.

El 58\% de estudiantes se encuentra en el rango intermedio, en otras palabras, existe un desarrollo hacia la actividad física y que no siempre se lo realiza dentro de la población, esto se debe ante lo mencionado por Claros et al. (2011) " A lo largo de los siglos la actividad física es una promoción de la salud que contribuye a construir resultados para el mejoramiento de una buena calidad de vida, ya que, esta ayuda a prevenir la obesidad •* (pp. 104-105). Cabe destacar que en la actualidad las personas que están en un rango intermedio optan por tomar acciones de promoción de actividad física teniendo en cuenta, que existen varios factores influyentes dentro de la realización de actividad física, como por ejemplo la falta de ganas, motivación o tiempo.

El 22\% de estudiantes se encuentra en el rango óptimo por lo cual infiere que presenta un buen desarrollo por la realización de actividad física, según William Ramírez (2004) 'la actividad física es un elemento que se ajusta a las necesidades de las personas ya que esta reduce en un gran porcentaje el estado de depresión y de ansiedad“ (pp. 67-75). Por tanto, la actividad física está presente de diversas formas, es necesario que las personas opten por un propósito de mejora hacia la estructura corporal llegando así a la realización de ejercicio físico que aportan a su salud.

\section{CONCLUSIÓN O CONSIDERACIONES FINALES}

De acuerdo a la revisión de la literatura, el sedentarismo repercute directamente en la vida de los estudiantes, afecta su salud física y mental, es decir, ser sedentario conlleva la aparición de factores de riesgos como enfermedades cardiovasculares, problemas de relaciones sociales, de salud mental, trastornos psicológicos e incluso alteraciones en el desarrollo psicomotor; por tal motivo, realizar actividad física moderada y con regularidad evitará la aparición de factores riesgosos; un joven universitario que 
mantenga una vida saludable y adecuada actividad física tendrá un correcto rendimiento académico, dado que no presenta afectaciones en su salud.

A partir de los resultados obtenidos de la encuesta aplicada a los estudiantes de la Carrera de Educación Inicial, un $22 \%$ de la muestra refleja que la organización de su tiempo libre no se orienta al desarrollo de actividades físicas; por lo que se propone, implementar Talleres con actividades recreativas donde se reflexione sobre la importancia de practicar el deporte para mejorar los hábitos de salud.

En la investigación se evidencia que, el total de muestra de estudiantes universitarios tiene interés en realizar la actividad física a pesar de las limitaciones cotidianas y académicas que reducen su tiempo, por ende, es necesario diseñar programas curriculares orientados al desarrollo de dichas actividades donde se informe sobre los beneficios y aportes que generan en su vida, a su vez, psicoeducar acerca de las enfermedades que produce el sedentarismo.

Es necesario resaltar que, existe un rango intermedio en el desarrollo de la actividad física, existiendo un $58 \%$ de estudiantes que optan por su realización por lo que es necesario que la Institución universitaria motive a los educandos a la práctica continúa del deporte, así mismo, cree espacios donde los estudiantes puedan realizar dicha actividad.

\section{LISTA DE REFERENCIAS}

Álvarez Pérez, P., Hernández Álvarez, A., \& López Aguilar, D. (2014). Análisis de la compleja relación entre los estudios universitarios y la práctica deportiva de alto rendimiento. Revista de la educación superior, 43(169), 69-87. http://publicaciones.anuies.mx/pdfs/revista/Revista169_S1A4ES.pdf

Bastos, A. D. A., Boto, R. G., González, O. M., \& del Valle, A. S. (2005). Obesidad, nutrición y actividad física. Revista Internacional de Medicina y Ciencias de la Actividad Física y del Deporte/International Journal of Medicine and Science of Physical Activity and 140-152. http://cdeporte.rediris.es/revista/revista18/artobesidad11.htm

Cala, O. C., \& Navarro, Y. B. (2011). La actividad física: un aporte para la salud. Lecturas: Educación física y deportes, (159), 5. https://dialnet.unirioja.es/servlet/articulo?codigo $=4684607$ 
Claros, V. J. A., Álvarez, C. V., Cuellar, C. S., \& Mora, M. L. A. (2011). Actividad física: estrategia de promoción de la salud. Hacia la Promoción de la Salud, 16(1), 202218. http://www.scielo.org.co/pdf/hpsal/v16n1/v16n1a14.pdf

Cvetkovic Vega, A., Maguiña, J. L., Soto, A., Lama, V. J., \& Correa L. L. E. (2021). Cross-sectional studies. Revista de la Facultad de Medicina Humana, 21(1), 164170. https://doi.org/10.25176/RFMH.v21i1.3069

Domínguez, D., Rodas, G. \& Ferrer, E. (2020). Actividad física y salud. Clínic Barcelona Hospital Universitario. https://www.clinicbarcelona.org/asistencia/cuida-tusalud/actividad-fisica-y-salud/definicion

Escalante, Y. (2011). Actividad física, ejercicio físico y condición física en el ámbito de la salud pública. Revista Española de la Salud Pública, 85(4), 325-328. https://scielo.isciii.es/pdf/resp/v85n4/01_editorial.pdf

Escobar, P. J., \& Martínez, A. (2008). Validez de contenido y juicio de expertos: Una aproximación a su utilización. Avances en Medición, 6, 27-36. http://www.scielo.org.mx/scielo.php?script=sci_arttext\&pid=S1665$\underline{61802017000300042}$

Escuela Nacional de Salud y Nutrición. (2013). Actividad física y sedentarismo. ENSANUT-ECU, 79-82. https://www.ecuadorencifras.gob.ec/documentos/webinec/Estadisticas_Sociales/ENSANUT/Publicacion\%20ENSANUT\%2020112013\%20tomo\%201.pdf

Gao, Z., Lee, J., Pope, Z., \& Zhang, D. (2016). Effect of active videogames on underserved children's classroom behaviors, effort, and fitness. Games for Health Journal, 318-324. https://experts.umn.edu/en/publications/effect-of-activevideogames-on-underserved-childrens-classroom-be

Hellin Gómez, P. (2003). Hábitos físico-deportivos en la Región de Murcia: implicaciones para la elaboración del currículum en el ciclo formativo de actividades físico-deportivas. Tesisenred.net. https://doi.org/9788469133521 https://www.tesisenred.net/handle/10803/10788

Herrera, G. E., Brocal, P. D., Mármol, D. J. S., \& Dorantes, J. M. R. (2012). Relación entre actividad física, depresión y ansiedad en adolescentes. Cuadernos de Psicología del Deporte, 12(2), 31-38. https://revistas.um.es/cpd/article/view/177731 
Leiva, A. M., Martínez, M. A., Montero, C. C., Salas, C., Ramírez, C. R., Díaz, M. X., Aguilar, F. N., \& Celis, M. C. (2017). El sedentarismo se asocia a un incremento de factores de riesgo cardiovascular y metabólicos independiente de los niveles de actividad física. Revista Médica de Chile, 145(6), 458-467. https://scielo.conicyt.cl/pdf/rmc/v145n4/art06.pdf

Márquez, R. S., Rodríguez, O. J., \& De Abajo Ole, S. (2006). Sedentarismo y salud: Efectos beneficiosos de la actividad física. Apuntes Educación física y deportes, $12-24$.

https://www.um.es/innova/OCW/educacion_fisica_y_salud/efs2012/lecturas/083 _012-024ES.pdf

Navarrete, M. P., Parodi, G. J., Vega, G. E., Pareja, C. A., \& Benites, A. J. (2019). Factores asociados al sedentarismo en jóvenes estudiantes de educación superior. Perú, 2017. Horizonte Médico-ResearchGate, 46-52. http://www.scielo.org.pe/scielo.php?script=sci_arttext\&pid=S1727558X2019000100008

Organización Mundial de la Salud. (2020). Cada movimiento cuenta para mejorar la salud. $\quad$ https://www.who.int/es/news/item/25-11-2020-every-move-countstowards-better-health-says-who

Oviedo, H. C., \& Arias, A. C. (2005). Aproximación al uso del coeficiente alfa de Cronbach. Revista colombiana de psiquiatría, 34(4), 572-580. https://www.redalyc.org/pdf/806/80634409.pdf

Perea, C. A., López N. G., Perea M. A., Reyes G. U., Lagunes, S., Ríos G. P., De La Paz Morales, C. (2019). Importancia de la Actividad Física. Médico-Científica de la $\begin{array}{llll}\text { Secretaría de } & \text { Salud }\end{array}$ https://www.medigraphic.com/pdfs/saljalisco/sj-2019/sj192h.pdf

Práxedes, Alba; Sevil, Javier; Moreno, Alberto; del Villar, Fernando; García-González, L. (2016). Niveles de Actividad Fisica. Revista Iberoamerica de LPsicología Del $\begin{array}{lllll}\text { Ejercicio } & y & \text { Deporte, } & 11,\end{array}$ https://www.redalyc.org/pdf/3111/311143051014.pdf

Ramírez, S. V. (2004). El impacto de la actividad física y el deporte sobre la salud, la cognición, la socialización y el rendimiento académico: Una revisión teórica. Scielo, 67-75. http://www.scielo.org.co/pdf/res/n18/n18a08.pdf 
Real Academia Española. (2020). Diccionario de la lengua española. (Edición del tricentenario). https://dle.rae.es/sedentario?m=form

Rivera, T. J., Cedillo, R. L., Pérez, N. J, Flores, C., B. \& Aguilar, E. R. (2018). Uso de tecnologías, sedentarismo y actividad física en estudiantes universitarios. Revista $\begin{array}{llll}\text { Iberoamericana de } \quad \text { Ciencias, } & \text { 17-23. }\end{array}$ http://www.reibci.org/publicados/2018/feb/2600103.pdf

Sautu, R., Boniolo, P., Dalle, P., \& Elbert, R. (2005). Manual de metodología: construcción del marco teórico, formulación de los objetivos y elección de la metodología.

http://biblioteca.clacso.edu.ar/gsdl/collect/clacso/index/assoc/D1532.dir/sautu2.p $\underline{\mathrm{df}}$

Tamayo y Tamayo, M. (2004). El proceso de la investigación científica incluye evaluación y administración de proyectos de investigación. Limusa, 37-90. https://www.gob.mx/cms/uploads/attachment/file/227860/El_proceso_de_la_in vestigaci_n_cient_fica_Mario_Tamayo.pdf

Vélez, Á. C., Vidarte, C. J. \& Parra, S. J. (2014). Niveles de sedentarismo en población entre 18 y 60 años en Manizales, Pereira y Armenia, Colombia. Análisis multivariado. $\quad$ Aquichan, 303-315. http://www.scielo.org.co/pdf/aqui/v14n3/v14n3a03.pdf 